\title{
Anthecularin, A Novel Sesquiterpene Lactone from Anthemis auriculata With Antiprotozoal Activity
}

Anastasia Karioti, ${ }^{* \dagger}$ Helen Skaltsa, ${ }^{\dagger}$ Anthony Linden, ${ }^{\ddagger}$ Remo Perozzo,${ }^{\S}$ Reto Brun, ${ }^{\text {II }}$ and Deniz Tasdemir ${ }^{*, *}$

${ }^{\dagger}$ Department of Pharmacognosy and Chemistry of Natural Products, School of Pharmacy, University of Athens, 157 71, Athens, Greece

${ }^{\ddagger}$ Institute of Organic Chemistry, University of Zurich, 8057 Zürich, Switzerland

${ }^{\S}$ School of Pharmaceutical Sciences, University of Geneva, 1211 Geneva 4, Switzerland

${ }^{I I}$ Department of Medical Parasitology, Swiss Tropical Institute, 4002 Basel, Switzerland

${ }^{¥}$ Centre for Pharmacognosy and Phytotherapy, University of London, London WCIN 1AX, UK

deniz.tasdemir@pharmacy.ac.uk

\section{Supporting Information-2}

(Continuation)

Page

3. Heteronuclear 2D NMR data of the new compound, anthecularin (1)

Figure S7. HSQC spectrum of anthecularin (1) in $\mathrm{CDCl}_{3}$

Figure S8. HSQC-TOCSY spectrum of anthecularin (1) in $\mathrm{CDCl}_{3} \quad \mathrm{~S} 13$

Figure S9. HMBC spectrum of anthecularin (1) in $\mathrm{CDCl}_{3}$

4. MS data of the new compound, anthecularin (1)

Figure S10. LREIMS spectrum of anthecularin (1)

Figure S11. HREIMS data of anthecularin (1)

5. X-ray crystallography, experimental and crystal data for anthecularin (1)

Experimental

Table 1. Crystallographic Data

Table 2. Fractional atomic coordinates and equivalent isotropic displacement

parameters $\left(\AA^{2}\right)$ with standard uncertainties in parentheses

Table 3. Bond lengths $(\AA)$ with standard uncertainties in parentheses

Table 4. Bond angles $\left(^{\circ}\right)$ with standard uncertainties in parentheses

Table 5. Torsion angles $\left(^{\circ}\right)$ with standard uncertainties in parentheses

Table 6. Anisotropic atomic displacement parameters $\left(\AA^{2}\right)$ 
3. Heteronuclear 2D NMR data of the new compound, anthecularin (1)

Figure S7. HSQC spectrum of anthecularin (1) in $\mathrm{CDCl}_{3}$

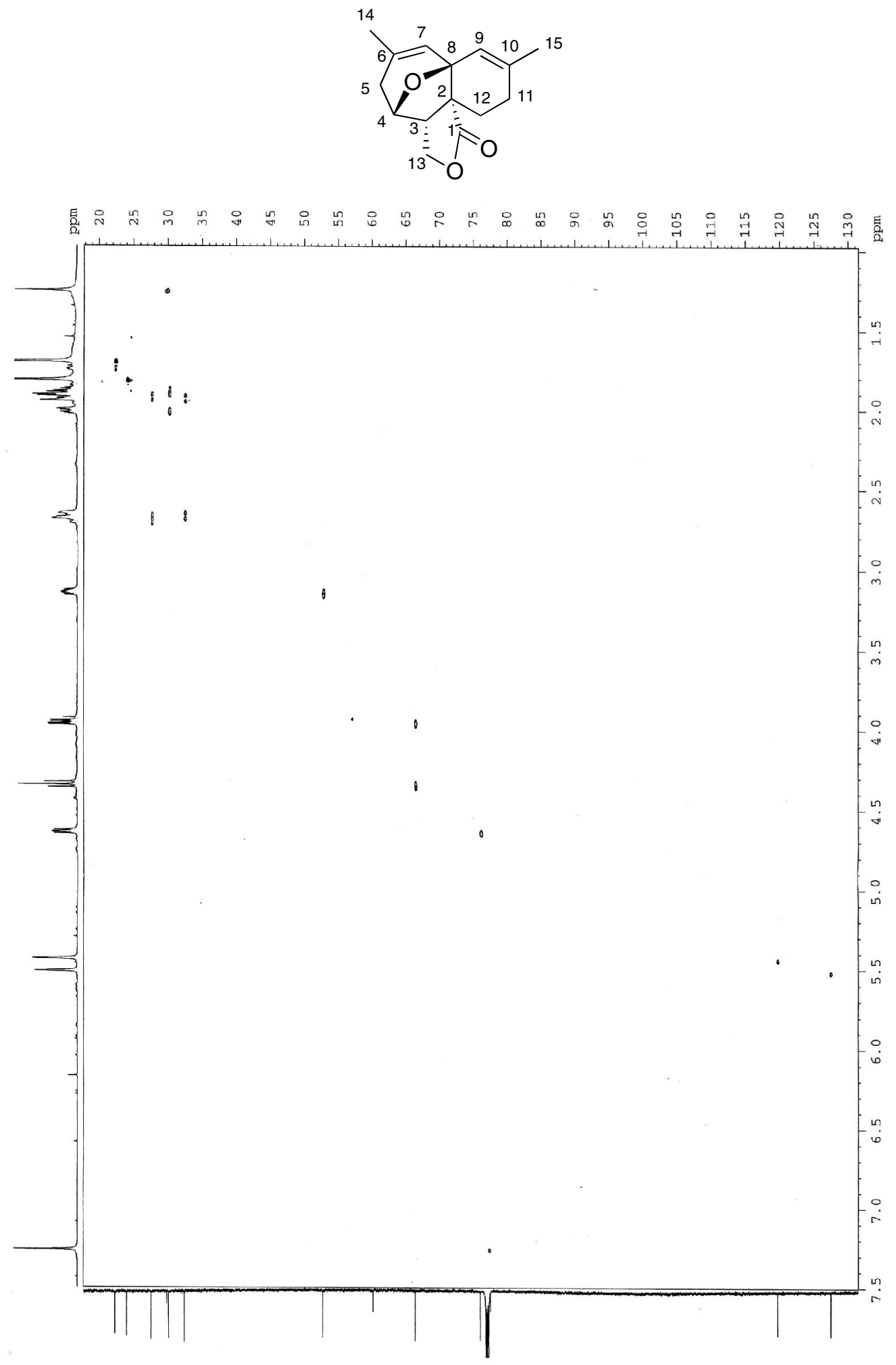


Figure S8. HSQC-TOCSY spectrum of anthecularin (1) in $\mathrm{CDCl}_{3}$

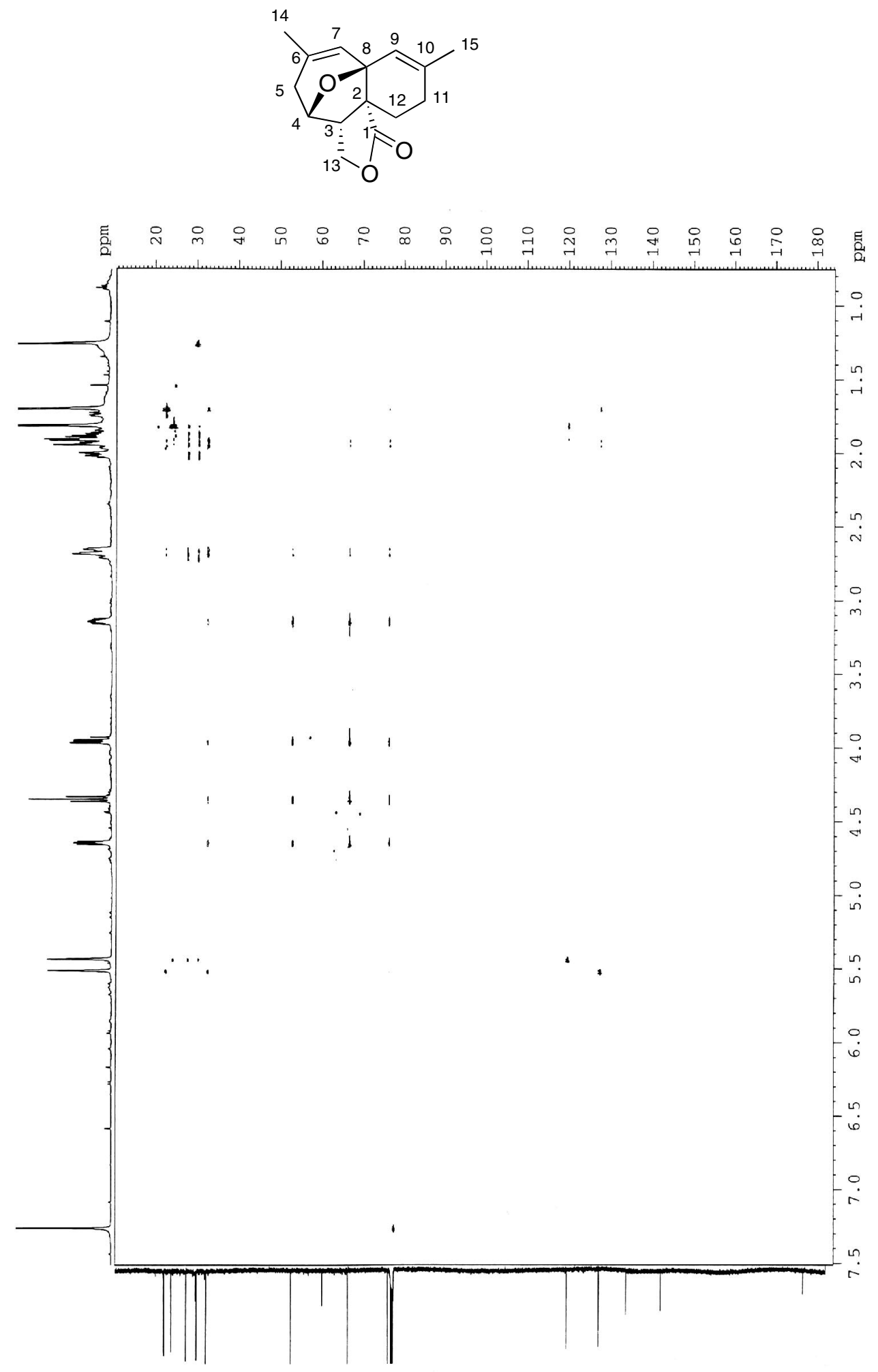


Figure S9. HMBC spectrum of anthecularin (1) in $\mathrm{CDCl}_{3}$
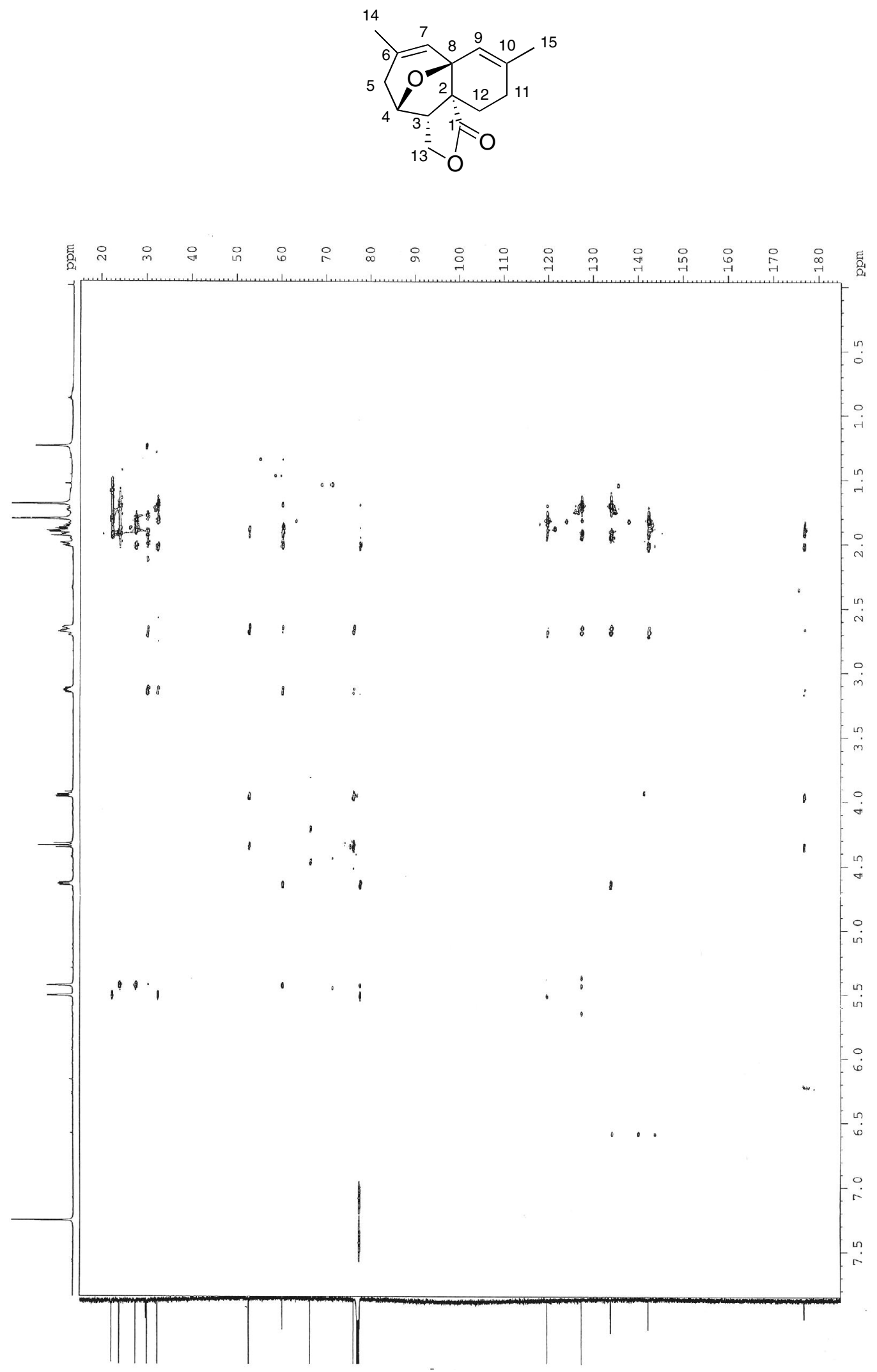
4. MS data of the new compound, anthecularin (1)

Figure S10. LREIMS spectrum of anthecularin (1)
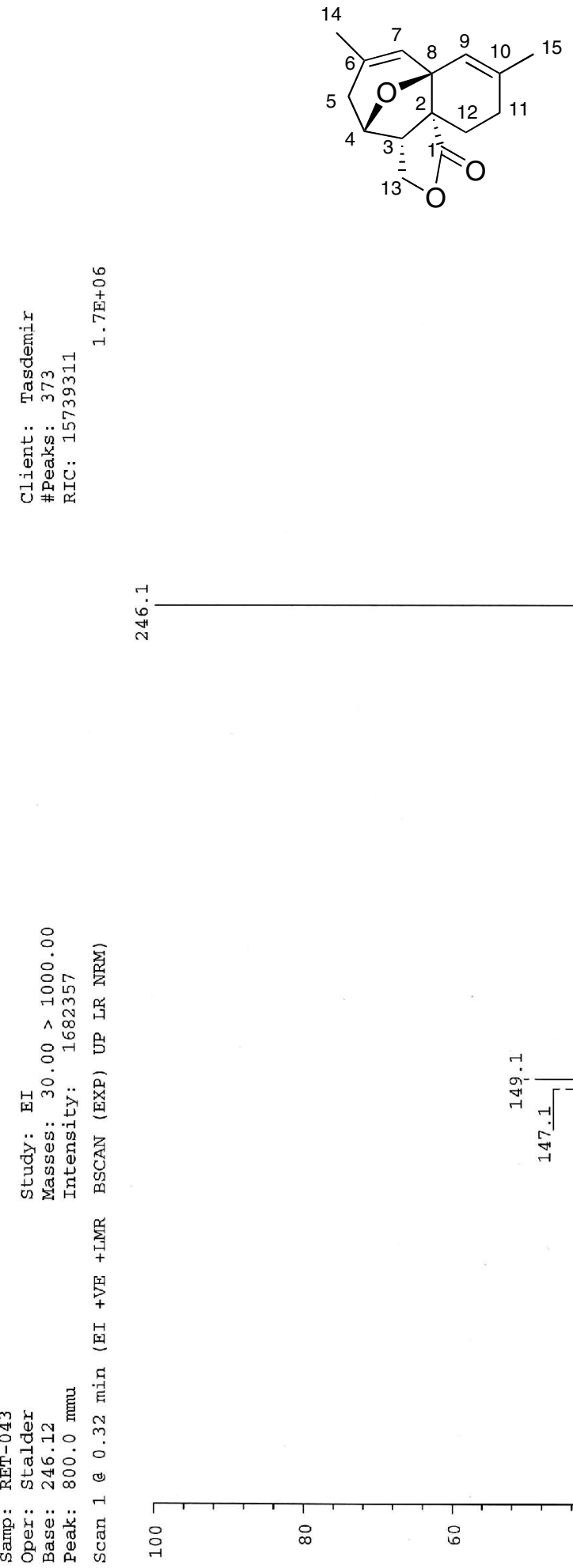

:

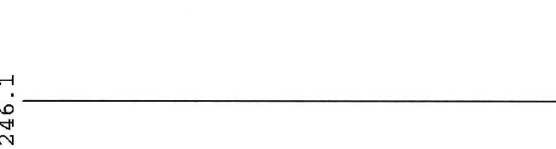

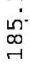
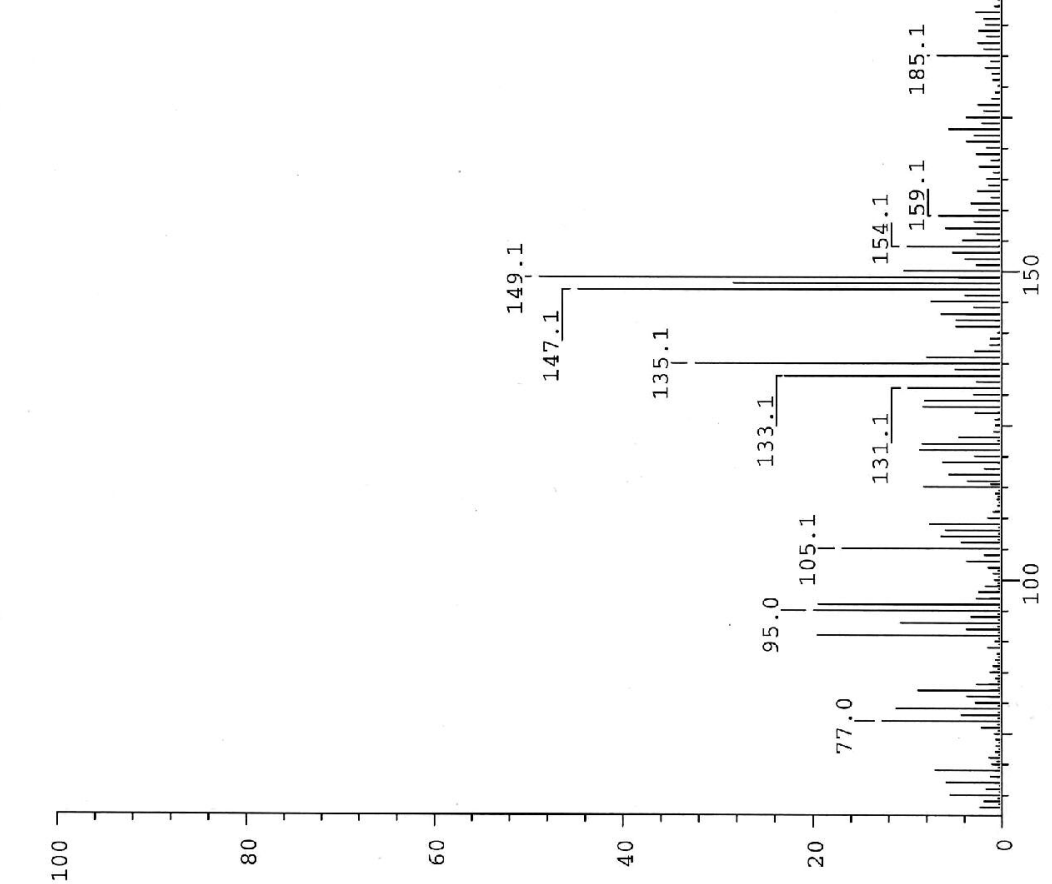
Figure S11. HREIMS data of anthecularin (1)
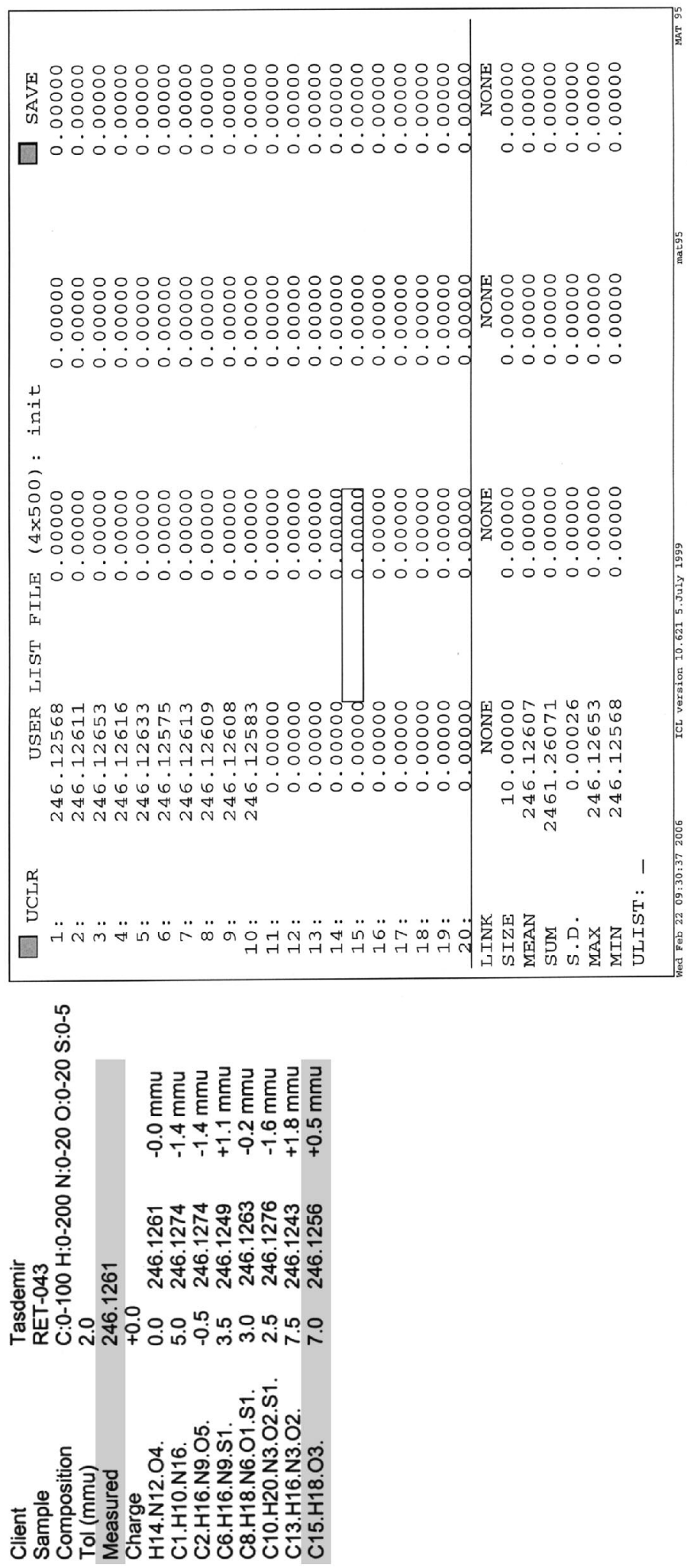


\section{X-ray crystallography, experimental and crystal data for anthecularin (1)}

\section{EXPERIMENTAL}

A crystal of $\mathrm{C}_{15} \mathrm{H}_{18} \mathrm{O}_{3}$, obtained from EtOAc / hexane (1:10), was mounted on a glass fibre and used for a low-temperature X-ray structure determination. All measurements were made on a Nonius KappaCCD area-detector diffractometer ${ }^{2}$ using graphite-monochromated Mo $K \alpha$ radiation $(\lambda=0.71073 \AA)$ and an Oxford Cryosystems Cryostream 700 cooler. The unit cell constants and an orientation matrix for data collection were obtained from a least-squares refinement of the setting angles of 1508 reflections in the range $4^{\circ}<2 \theta<55^{\circ}$. The mosaicity was $0.453(2)^{\circ}$. A total of 335 frames were collected using $\phi$ and $\omega$ scans with $\kappa$ offsets, 60 seconds exposure time and a rotation angle of $2.0^{\circ}$ per frame, and a crystal-detector distance of $30.0 \mathrm{~mm}$.

Data reduction was performed with HKL Denzo and Scalepack ${ }^{3}$. The intensities were corrected for Lorentz and polarization effects, but not for absorption. The space group was determined from the systematic absences, packing considerations, a statistical analysis of intensity distribution, and the successful solution and refinement of the structure. Equivalent reflections were merged. The data collection and refinement parameters are given in Table 1. A view of the molecule is shown in the Figure.

The structure was solved by direct methods using $\operatorname{SIR} 92^{4}$, which revealed the positions of all nonhydrogen atoms. The non-hydrogen atoms were refined anisotropically. All of the H-atoms were placed in geometrically calculated positions and refined by using a riding model where each $\mathrm{H}$-atom was assigned a fixed isotropic displacement parameter with a value equal to $1.2 \mathrm{U}_{\mathrm{eq}}$ of its parent atom $(1.5 \mathrm{U}$ eq for the methyl groups). The refinement of the structure was carried out on $F^{2}$ by using full-matrix least-squares procedures, which minimised the function $\Sigma w\left(F_{\mathrm{O}}{ }^{2}-F_{\mathrm{c}}{ }^{2}\right)^{2}$. The weighting scheme was based on counting statistics and included a factor to downweight the intense reflections. Plots of $\Sigma w\left(F_{\mathrm{O}}^{2}-F_{\mathrm{c}}^{2}\right)^{2}$ versus $F_{\mathrm{C}} / F_{\mathrm{C}}(\max )$ and resolution showed no unusual trends. A correction for secondary extinction was applied. One reflection, whose intensity was considered to be an extreme outlier, was omitted from the final refinement.

Neutral atom scattering factors for non-hydrogen atoms were taken from Maslen, Fox and $\mathrm{O}^{\prime K}$ Keefe $5 \mathrm{a}$, and the scattering factors for $\mathrm{H}$-atoms were taken from Stewart, Davidson and Simpson 6 . Anomalous dispersion effects were included in $F_{\mathrm{c}}{ }^{7}$; the values for $f^{\prime}$ and $f^{\prime \prime}$ were those of Creagh and McAuley $5 \mathrm{~b}$. The values of the mass attenuation coefficients are those of Creagh and Hubbel $5 \mathrm{c}$. The SHELXL97 program $^{8}$ was used for all calculations. 


\section{REFERENCES}

(1) C.K. Johnson, ORTEPII, Report ORNL-5138, Oak Ridge National Laboratory, Oak Ridge, Tennessee, 1976.

(2) R. Hooft, KappaCCD Collect Software, Nonius BV, Delft, The Netherlands, 1999.

(3) Z. Otwinowski, W. Minor, in Methods in Enzymology, Vol. 276, Macromolecular Crystallography, Part A, Eds. C.W. Carter Jr., R.M. Sweet, Academic Press, New York, 1997, pp. 307-326.

(4) A. Altomare, G. Cascarano, C. Giacovazzo, A. Guagliardi, M.C. Burla, G. Polidori, M. Camalli, SIR92, J. Appl. Crystallogr. 1994, 27, 435.

(5) a) E.N. Maslen, A.G. Fox, M.A. O'Keefe, in 'International Tables for Crystallography', Ed. A.J.C. Wilson, Kluwer Academic Publishers, Dordrecht, 1992, Vol. C, Table 6.1.1.1, pp. 477-486; b) D.C. Creagh, W.J. McAuley, ibid. Table 4.2.6.8, pp. 219-222; c) D.C. Creagh, J.H. Hubbell, ibid. Table 4.2.4.3, pp. 200-206.

(6) R.F. Stewart, E.R. Davidson, W.T. Simpson, J. Chem. Phys. 1965, 42, 3175-3187.

(7) J.A. Ibers, W.C. Hamilton, Acta Crystallogr. 1964, 17, 781-782.

(8) G.M. Sheldrick, SHELXL97, Program for the Refinement of Crystal Structures, University of Göttingen, Germany, 1997. 
Table 1. Crystallographic Data

Crystallised from

Empirical formula

Formula weight $\left[\mathrm{g} \mathrm{mol}^{-1}\right.$ ]

Crystal colour, habit

Crystal dimensions [mm]

Temperature $[\mathrm{K}]$

Crystal system

Space group

$Z$

Reflections for cell determination

$2 \theta$ range for cell determination $\left[{ }^{\circ}\right]$

Unit cell parameters $\quad a[\AA]$

$b[\AA]$

$c[\AA]$

$\alpha\left[{ }^{\circ}\right]$

$\beta\left[^{\circ}\right]$

$\gamma\left[{ }^{\circ}\right]$

$F(000)$

$V\left[\AA^{3}\right]$

$D_{x}\left[\mathrm{~g} \mathrm{~cm}^{-3}\right]$

$\mu(\mathrm{Mo} K \alpha)\left[\mathrm{mm}^{-1}\right]$

Scan type

$2 \theta_{(\max )}\left[{ }^{\circ}\right]$

Total reflections measured

Symmetry independent reflections

$R_{\text {int }}$

Reflections with $I>2 \sigma(I)$

Reflections used in refinement

Parameters refined; restraints

Final $\quad R(F)[I>2 \sigma(I)$ reflections $]$

$$
w R\left(F^{2}\right) \text { (all data) }
$$

Weights:

Goodness of fit

Secondary extinction coefficient

Final $\Delta_{\max } / \sigma$

$\Delta \rho(\max ; \min )\left[\mathrm{e} \AA^{-3}\right]$

$\sigma\left(d_{(\mathrm{C}-\mathrm{C})}\right)[\AA]$
EtOAc / hexane (1:10)

$\mathrm{C}_{15} \mathrm{H}_{18} \mathrm{O}_{3}$

246.30

colourless, tablet

$0.08 \times 0.17 \times 0.17$

160(1)

monoclinic

P21 (\#4)

2

1508

4-55

$7.0010(2)$

$12.2264(3)$

$7.3355(2)$

90

92.634(2)

90

627.23(3)

264

1.304

0.0895

$\phi$ and $\omega$

55

14891

1503

0.057

1403

1502

166; 1

0.0432

0.1037

$w=\left[\sigma^{2}\left(F_{\mathrm{O}}{ }^{2}\right)+(0.0648 P)^{2}+0.0324 P\right]^{-1}$ where $P=\left(F_{\mathrm{O}}{ }^{2}+2 F_{\mathrm{c}}{ }^{2}\right) / 3$

1.123

$0.46(4)$

0.001

$0.30 ;-0.23$

0.003 
TABLE 2. Fractional atomic coordinates and equivalent isotropic displacement parameters $\left(\AA^{2}\right)$ with standard uncertainties in parentheses.

${ }^{*} \mathrm{U}_{\mathrm{eq}}$ is defined as one third of the trace of the orthogonalized $\mathrm{U}^{\mathrm{ij}}$ tensor.

$\begin{array}{lllll}\text { ATOM } & \mathrm{x} & \mathrm{Y} & \mathrm{z} & \mathrm{U}_{\mathrm{eq}}{ }^{*} \\ \text { O(1) } & 1.0656(2) & 0.3011(2) & 0.6259(2) & 0.0459(5) \\ \text { O(2) } & 0.7900(3) & 0.3446(2) & 0.7470(2) & 0.0453(5) \\ \text { O(3) } & 0.5648(2) & 0.1289(1) & 0.3518(2) & 0.0311(4) \\ \text { C(1) } & 0.8935(3) & 0.3052(2) & 0.6107(3) & 0.0350(5) \\ \text { C(2) } & 0.7644(3) & 0.2707(2) & 0.4492(3) & 0.0292(5) \\ \text { C(3) } & 0.5594(3) & 0.2966(2) & 0.5071(3) & 0.0337(5) \\ \text { C(4) } & 0.4506(3) & 0.1865(2) & 0.4797(3) & 0.0334(5) \\ \text { C(5) } & 0.4346(3) & 0.1158(2) & 0.6478(3) & 0.0365(5) \\ \text { C(6) } & 0.6278(3) & 0.0729(2) & 0.7135(3) & 0.0303(5) \\ \text { C (7) } & 0.7777(3) & 0.0869(2) & 0.6101(3) & 0.0279(4) \\ \text { C(8) } & 0.7587(3) & 0.1441(2) & 0.4266(3) & 0.0261(4) \\ \text { C(9) } & 0.8956(3) & 0.0990(2) & 0.2953(3) & 0.0293(5) \\ \text { C(10) } & 1.0069(3) & 0.1597(2) & 0.1930(3) & 0.0304(5) \\ \text { C(11) } & 1.0004(3) & 0.2826(2) & 0.1942(3) & 0.0354(5) \\ \text { C(12) } & 0.8165(3) & 0.3253(2) & 0.2705(3) & 0.0355(5) \\ \text { C(13) } & 0.5865(4) & 0.3458(2) & 0.6984(4) & 0.0440(6) \\ \text { C(14) } & 0.6385(4) & 0.0145(2) & 0.8942(3) & 0.0386(5) \\ \text { C(15) } & 1.1449(3) & 0.1096(2) & 0.0654(3) & 0.0397(6)\end{array}$


TABLE 3. Bond lengths $(\AA)$ with standard uncertainties in parentheses.

$\begin{array}{llllll}\mathrm{O}(1) & -\mathrm{C}(1) & 1.206(3) & \mathrm{C}(4) & -\mathrm{C}(5) & 1.515(3) \\ \mathrm{O}(2) & -\mathrm{C}(1) & 1.350(3) & \mathrm{C}(5) & -\mathrm{C}(6) & 1.509(3) \\ \mathrm{O}(2) & -\mathrm{C}(13) & 1.453(3) & \mathrm{C}(6) & -\mathrm{C}(7) & 1.334(3) \\ \mathrm{O}(3) & -\mathrm{C}(4) & 1.444(3) & \mathrm{C}(6) & -\mathrm{C}(14) & 1.504(3) \\ \mathrm{O}(3) & -\mathrm{C}(8) & 1.453(2) & \mathrm{C}(7) & -\mathrm{C}(8) & 1.517(3) \\ \mathrm{C}(1) & -\mathrm{C}(2) & 1.517(3) & \mathrm{C}(8) & -\mathrm{C}(9) & 1.495(3) \\ \mathrm{C}(2) & -\mathrm{C}(12) & 1.530(3) & \mathrm{C}(9) & -\mathrm{C}(10) & 1.332(3) \\ \mathrm{C}(2) & -\mathrm{C}(3) & 1.549(3) & \mathrm{C}(10) & -\mathrm{C}(11) & 1.503(3) \\ \mathrm{C}(2) & -\mathrm{C}(8) & 1.557(3) & \mathrm{C}(10) & -\mathrm{C}(15) & 1.506(3) \\ \mathrm{C}(3) & -\mathrm{C}(13) & 1.530(3) & \mathrm{C}(11) & -\mathrm{C}(12) & 1.520(3) \\ \mathrm{C}(3) & -\mathrm{C}(4) & 1.555(3) & & & \end{array}$

TABLE 4. Bond angles $\left({ }^{\circ}\right)$ with standard uncertainties in parentheses.

$\begin{array}{llllllll}C(1) & -\mathrm{O}(2) & -\mathrm{C}(13) & 112.0(2) & \mathrm{C}(7) & -\mathrm{C}(6) & -\mathrm{C}(14) & 123.6(2) \\ \mathrm{C}(4) & -\mathrm{O}(3) & -\mathrm{C}(8) & 103.2(1) & \mathrm{C}(7) & -\mathrm{C}(6) & -\mathrm{C}(5) & 119.4(2) \\ \mathrm{O}(1) & -\mathrm{C}(1) & -\mathrm{O}(2) & 121.0(2) & \mathrm{C}(14) & -\mathrm{C}(6) & -\mathrm{C}(5) & 117.0(2) \\ \mathrm{O}(1) & -\mathrm{C}(1) & -\mathrm{C}(2) & 128.1(2) & \mathrm{C}(6) & -\mathrm{C}(7) & -\mathrm{C}(8) & 121.6(2) \\ \mathrm{O}(2) & -\mathrm{C}(1) & -\mathrm{C}(2) & 110.9(2) & \mathrm{O}(3) & -\mathrm{C}(8) & -\mathrm{C}(9) & 109.0(2) \\ \mathrm{C}(1) & -\mathrm{C}(2) & -\mathrm{C}(12) & 113.0(2) & \mathrm{O}(3) & -\mathrm{C}(8) & -\mathrm{C}(7) & 108.6(2) \\ \mathrm{C}(1) & -\mathrm{C}(2) & -\mathrm{C}(3) & 104.8(2) & \mathrm{C}(9) & -\mathrm{C}(8) & -\mathrm{C}(7) & 111.6(2) \\ \mathrm{C}(12) & -\mathrm{C}(2) & -\mathrm{C}(3) & 114.0(2) & \mathrm{O}(3) & -\mathrm{C}(8) & -\mathrm{C}(2) & 100.7(2) \\ \mathrm{C}(1) & -\mathrm{C}(2) & -\mathrm{C}(8) & 111.8(2) & \mathrm{C}(9) & -\mathrm{C}(8) & -\mathrm{C}(2) & 114.9(2) \\ \mathrm{C}(12) & -\mathrm{C}(2) & -\mathrm{C}(8) & 110.4(2) & \mathrm{C}(7) & -\mathrm{C}(8) & -\mathrm{C}(2) & 111.3(2) \\ \mathrm{C}(3) & -\mathrm{C}(2) & -\mathrm{C}(8) & 102.3(2) & \mathrm{C}(10) & -\mathrm{C}(9) & -\mathrm{C}(8) & 124.5(2) \\ \mathrm{C}(13) & -\mathrm{C}(3) & -\mathrm{C}(2) & 104.8(2) & \mathrm{C}(9) & -\mathrm{C}(10) & -\mathrm{C}(11) & 122.3(2) \\ \mathrm{C}(13) & -\mathrm{C}(3) & -\mathrm{C}(4) & 119.9(2) & \mathrm{C}(9) & -\mathrm{C}(10) & -\mathrm{C}(15) & 122.2(2) \\ \mathrm{C}(2) & -\mathrm{C}(3) & -\mathrm{C}(4) & 104.0(2) & \mathrm{C}(11) & -\mathrm{C}(10) & -\mathrm{C}(15) & 115.5(2) \\ \mathrm{O}(3) & -\mathrm{C}(4) & -\mathrm{C}(5) & 108.3(2) & \mathrm{C}(10) & -\mathrm{C}(11) & -\mathrm{C}(12) & 111.8(2) \\ \mathrm{O}(3) & -\mathrm{C}(4) & -\mathrm{C}(3) & 102.9(2) & \mathrm{C}(11) & -\mathrm{C}(12) & -\mathrm{C}(2) & 113.9(2) \\ \mathrm{C}(5) & -\mathrm{C}(4) & -\mathrm{C}(3) & 116.3(2) & \mathrm{O}(2) & -\mathrm{C}(13) & -\mathrm{C}(3) & 107.5(2) \\ \mathrm{C}(6) & -\mathrm{C}(5) & -\mathrm{C}(4) & 111.1(2) & & & & \end{array}$


TABLE 5. Torsion angles $\left({ }^{\circ}\right)$ with standard uncertainties in parentheses.

\begin{tabular}{|c|c|c|c|c|c|c|c|c|c|}
\hline$C(13)$ & $-O(2)$ & $-C(1)$ & $-O(1)$ & $178.1(2)$ & $C(4)$ & $-O(3)$ & $-C(8)$ & $-C(2)$ & $-52.4(2)$ \\
\hline$C(13)$ & $-O(2)$ & $-C(1)$ & $-C(2)$ & $-1.8(3)$ & $C(6)$ & $-C(7)$ & $-C(8)$ & $-O(3)$ & $-27.6(3)$ \\
\hline$O(1)$ & $-C(1)$ & $-C(2)$ & $-\mathrm{C}(12)$ & $-54.5(3)$ & $C(6)$ & $-C(7)$ & $-C(8)$ & $-C(9)$ & $-147.9(2)$ \\
\hline$O(2)$ & $-C(1)$ & $-C(2)$ & - C (12) & $125.3(2)$ & $C(6)$ & $-C(7)$ & $-C(8)$ & $-C(2)$ & $82.3(2)$ \\
\hline$O(1)$ & $-C(1)$ & $-C(2)$ & $-C(3)$ & $-179.2(2)$ & $\mathrm{C}(1)$ & $-C(2)$ & $-C(8)$ & $-O(3)$ & $148.1(2)$ \\
\hline$O(2)$ & $-C(1)$ & $-C(2)$ & $-C(3)$ & $0.6(2)$ & C (12) & $-C(2)$ & $-C(8)$ & $-O(3)$ & $-85.2(2)$ \\
\hline$O(1)$ & $-C(1)$ & $-C(2)$ & $-C(8)$ & $70.8(3)$ & $C(3)$ & $-C(2)$ & $-C(8)$ & $-O(3)$ & $36.5(2)$ \\
\hline$O(2)$ & $-C(1)$ & $-C(2)$ & $-C(8)$ & $-109.4(2)$ & $C(1)$ & $-C(2)$ & $-C(8)$ & $-\mathrm{C}(9)$ & $-95.0(2)$ \\
\hline$C(1)$ & $-C(2)$ & $-C(3)$ & $-C(13)$ & $0.6(2)$ & C (12) & $-C(2)$ & $-C(8)$ & $-C(9)$ & $31.8(2)$ \\
\hline C (12) & $-C(2)$ & $-C(3)$ & $-C(13)$ & $-123.4(2)$ & $C(3)$ & $-C(2)$ & $-C(8)$ & $-C(9)$ & $153.4(2)$ \\
\hline$C(8)$ & $-C(2)$ & $-C(3)$ & $-C(13)$ & $117.4(2)$ & $C(1)$ & $-C(2)$ & $-C(8)$ & $-C(7)$ & $33.1(2)$ \\
\hline$C(1)$ & $-C(2)$ & $-C(3)$ & $-C(4)$ & $-126.0(2)$ & C (12) & $-C(2)$ & $-C(8)$ & $-C(7)$ & $159.9(2)$ \\
\hline $\mathrm{C}(12)$ & $-C(2)$ & $-C(3)$ & $-C(4)$ & $110.0(2)$ & $C(3)$ & $-C(2)$ & $-C(8)$ & $-C(7)$ & $-78.5(2)$ \\
\hline $\mathrm{C}(8)$ & $-C(2)$ & $-C(3)$ & $-C(4)$ & $-9.2(2)$ & $O(3)$ & $-C(8)$ & $-C(9)$ & $-C(10)$ & $107.4(2)$ \\
\hline$C(8)$ & $-O(3)$ & $-C(4)$ & $-C(5)$ & $-77.2(2)$ & $C(7)$ & $-C(8)$ & $-\mathrm{C}(9)$ & $-C(10)$ & $-132.6(2)$ \\
\hline$C(8)$ & $-O(3)$ & $-C(4)$ & $-C(3)$ & $46.5(2)$ & $C(2)$ & $-C(8)$ & $-C(9)$ & $-C(10)$ & $-4.7(3)$ \\
\hline$C(13)$ & $-C(3)$ & $-C(4)$ & $-O(3)$ & $-137.9(2)$ & $C(8)$ & $-C(9)$ & $-C(10)$ & $-C(11)$ & $-2.3(3)$ \\
\hline$C(2)$ & $-C(3)$ & $-C(4)$ & $-O(3)$ & $-21.4(2)$ & $C(8)$ & $-C(9)$ & $-C(10)$ & $-C(15)$ & $178.9(2)$ \\
\hline C (13) & $-C(3)$ & $-C(4)$ & $-C(5)$ & $-19.7(3)$ & $C(9)$ & $-C(10)$ & $-C(11)$ & $-C(12)$ & $-18.9(3)$ \\
\hline$C(2)$ & $-C(3)$ & $-C(4)$ & $-C(5)$ & $96.8(2)$ & $C(15)$ & $-C(10)$ & $-C(11)$ & $-C(12)$ & $160.0(2)$ \\
\hline$O(3)$ & $-C(4)$ & $-C(5)$ & $-C(6)$ & $48.2(2)$ & $C(10)$ & $-C(11)$ & $-\mathrm{C}(12)$ & $-C(2)$ & $47.5(3)$ \\
\hline$C(3)$ & $-C(4)$ & $-C(5)$ & $-C(6)$ & $-67.0(2)$ & $C(1)$ & $-C(2)$ & $-C(12)$ & $-C(11)$ & $72.1(2)$ \\
\hline $\mathrm{C}(4)$ & $-C(5)$ & $-C(6)$ & $-C(7)$ & $-9.2(3)$ & $C(3)$ & $-C(2)$ & $-\mathrm{C}(12)$ & $-C(11)$ & $-168.5(2)$ \\
\hline$C(4)$ & $-C(5)$ & $-C(6)$ & $-\mathrm{C}(14)$ & $171.6(2)$ & $\mathrm{C}(8)$ & $-C(2)$ & $-C(12)$ & $-C(11)$ & $-54.0(3)$ \\
\hline C (14) & $-C(6)$ & $-C(7)$ & $-C(8)$ & $178.5(2)$ & $C(1)$ & $-O(2)$ & $-C(13)$ & $-C(3)$ & $2.1(3)$ \\
\hline$C(5)$ & $-C(6)$ & $-C(7)$ & $-C(8)$ & $-0.6(3)$ & $C(2)$ & $-C(3)$ & $-C(13)$ & $-O(2)$ & $-1.6(3)$ \\
\hline$C(4)$ & $-O(3)$ & $-C(8)$ & $-C(9)$ & $-173.6(2)$ & $\mathrm{C}(4)$ & $-C(3)$ & $-C(13)$ & $-O(2)$ & $114.5(2)$ \\
\hline C ( 4$)$ & $-O(3)$ & $-C(8)$ & $-C(7)$ & $64.6(2)$ & & & & & \\
\hline
\end{tabular}


TABLE 6. Anisotropic atomic displacement parameters $\left(\AA^{2}\right)$.

$\begin{array}{lllllll}\text { ATOM } & U^{11} & U^{22} & U^{33} & U^{23} & U^{13} & U^{12} \\ \text { O(1) } & 0.0395(9) & 0.044(1) & 0.053(1) & -0.0081(8) & -0.0086(8) & -0.0015(8) \\ \text { O(2) } & 0.055(1) & 0.0416(9) & 0.0390(9) & -0.0113(8) & 0.0018(7) & 0.0032(8) \\ \text { O(3) } & 0.0232(7) & 0.0401(8) & 0.0301(7) & -0.0050(6) & 0.0010(5) & 0.0008(6) \\ \text { C(1) } & 0.042(1) & 0.027(1) & 0.036(1) & -0.0021(9) & 0.0000(9) & 0.0002(9) \\ \text { C(2) } & 0.0261(9) & 0.030(1) & 0.031(1) & 0.0005(8) & 0.0029(8) & 0.0028(8) \\ \text { C(3) } & 0.033(1) & 0.034(1) & 0.035(1) & 0.0025(9) & 0.0070(8) & 0.0091(9) \\ \text { C(4) } & 0.0233(9) & 0.043(1) & 0.034(1) & -0.0013(9) & 0.0041(8) & 0.0049(9) \\ \text { C(5) } & 0.029(1) & 0.042(1) & 0.039(1) & 0.001(1) & 0.0088(8) & -0.001(1) \\ \text { C(6) } & 0.033(1) & 0.030(1) & 0.028(1) & -0.0021(8) & 0.0045(8) & -0.0024(9) \\ \text { C(7) } & 0.0277(9) & 0.028(1) & 0.0284(9) & -0.0022(8) & 0.0013(7) & 0.0016(8) \\ \text { C(8) } & 0.0198(8) & 0.029(1) & 0.030(1) & -0.0004(8) & 0.0005(7) & 0.0006(7) \\ \text { C(9) } & 0.027(1) & 0.032(1) & 0.028(1) & -0.0029(8) & 0.0010(8) & 0.0022(8) \\ \text { C(10) } & 0.0242(9) & 0.041(1) & 0.026(1) & 0.0005(9) & 0.0016(7) & 0.0030(8) \\ \text { C(11) } & 0.030(1) & 0.041(1) & 0.036(1) & 0.009(1) & 0.0063(8) & 0.0022(9) \\ \text { C(12) } & 0.036(1) & 0.033(1) & 0.038(1) & 0.0075(9) & 0.0060(9) & 0.0056(9) \\ \text { C(13) } & 0.049(1) & 0.041(1) & 0.043(1) & -0.007(1) & 0.014(1) & 0.005(1) \\ \text { C(14) } & 0.046(1) & 0.041(1) & 0.029(1) & 0.002(1) & 0.0062(9) & -0.005(1) \\ \text { C(15) } & 0.032(1) & 0.056(2) & 0.032(1) & -0.002(1) & 0.0091(8) & 0.005(1)\end{array}$

The anisotropic displacement parameter exponent takes the form:

$-2 \cdot{ }^{2}\left(h^{2} a * 2 U^{11}+k^{2} b * 2 U^{22}+\ldots+2 h k a * b^{*} U^{12}\right)$ 
TABLE 7. Hydrogen atom coordinates and displacement parameters.

$\begin{array}{lcccc}\text { ATOM } & \text { X } & y & z & U_{\text {iso }} \\ \text { H (3) } & 0.501 & 0.353 & 0.422 & 0.040 \\ \text { H (4) } & 0.320 & 0.201 & 0.423 & 0.040 \\ \text { H (51) } & 0.379 & 0.159 & 0.746 & 0.044 \\ \text { H (52) } & 0.348 & 0.053 & 0.619 & 0.044 \\ \text { H(7) } & 0.899 & 0.060 & 0.652 & 0.033 \\ \text { H(9) } & 0.904 & 0.022 & 0.284 & 0.035 \\ \text { H(111) } & 1.011 & 0.310 & 0.068 & 0.043 \\ \text { H(112) } & 1.111 & 0.311 & 0.269 & 0.043 \\ \text { H(121) } & 0.710 & 0.314 & 0.178 & 0.043 \\ \text { H (122) } & 0.829 & 0.405 & 0.291 & 0.043 \\ \text { H(131) } & 0.537 & 0.422 & 0.699 & 0.053 \\ \text { H(132) } & 0.516 & 0.302 & 0.787 & 0.053 \\ \text { H(141) } & 0.769 & -0.012 & 0.920 & 0.058 \\ \text { H(142) } & 0.550 & -0.048 & 0.890 & 0.058 \\ \text { H(143) } & 0.604 & 0.065 & 0.991 & 0.058 \\ \text { H(151) } & 1.144 & 0.030 & 0.079 & 0.060 \\ \text { H(152) } & 1.274 & 0.137 & 0.095 & 0.060 \\ \text { H(153) } & 1.107 & 0.129 & -0.061 & 0.060\end{array}$


Figure S12. X-ray crystal structure (ORTEP drawing) of anthecularin (1)

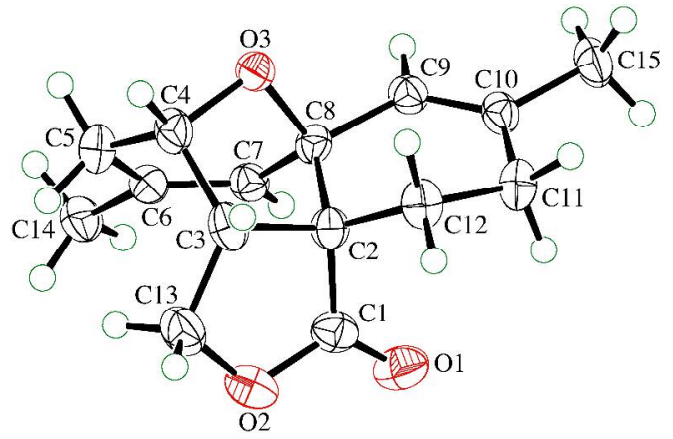

The molecular structure of anthecularin. Displacement ellipsoids are drawn at the $50 \%$ probability level. Hydrogen atoms have been drawn at an arbitrary size. 